\title{
Theoretical study on the thermal dissociation of FOX-7 promoted by $\mathrm{NO} 2$
}

\author{
Shuhui Yin ${ }^{1}$, Qiong Zhu ${ }^{1}$, Jianyong Liu $^{2}$, and Panwang Zhou ${ }^{3}$ \\ ${ }^{1}$ Dalian Maritime University \\ ${ }^{2}$ Dalian Institute of Chemical Physics \\ ${ }^{3}$ Shandong University
}

October 22, 2021

\begin{abstract}
1,1-diamino-2,2-dinitroethene (FOX-7) is a novel energetic material with high performance and low sensitivity. In order to deeply understand the reaction mechanism in the initiation "hot spots" of FOX-7 and reveal the growth mechanism of these initiation "hot spots" in the explosion process, the detailed mechanisms of bimolecular reaction of NO2 and FOX-7, as well as the subsequent reactions have been investigated by the quantum chemical calculations. The mechanism of NO2 and FOX-7 bimolecular reaction and the catalytic effect of NO2 were revealed by three key dissociation paths. It is demonstrated that the NO2 molecule plays an important role in promoting the decomposition of the FOX-7 molecule, and the main exothermic pathways were the reactions between oxidizing intermediates (NO, NO2), and reducing intermediates (CO, NH3).
\end{abstract}

\section{Hosted file}

Theoretical study on the thermal dissociation of FOX-7 promoted by N02.docx available at https://authorea.com/users/442338/articles/542628-theoretical-study-on-the-thermal-

dissociation-of-fox-7-promoted-by-no2 